\title{
An Application of Graph Theory in the Process of Mutual Debt Compensation
}

\section{Vladimír Gazda, Denis Horváth, Marcel Rešovský}

Technical University of Košice, Faculty of Economics; Němcovej 32, 04001

Košice, Slovakia, vladimir.gazda@tuke.sk

Pavol Jozef Šafárik University in Košice, Faculty of Science; Park Angelinum 9, 04001 Košice, Slovakia, denis.horvath@upjs.sk

Technical University of Košice, Faculty of Economics; Němcovej 32, 04001 Košice, Slovakia, marcel.resovsky@tuke.sk

Abstract: In our paper, the class of graph tractable problems of mutual debt compensation $(M D C)$ among firms is introduced. We demonstrate that the debt compensation is related to the optimization problems maximizing the circulation of the consecutive compensation in the digraph of debts. Each optimization problem is firstly formulated by linear programming methods. It is subsequently redeveloped in order to apply the more efficient Klein's cycle-cancelling algorithm. The class of the formulated compensation problems consists of the following models: (i) the model maximizing the returns of the MDC organizer; (ii) the model of profitable subsidies and (iii) the model minimizing the claims exposition of the MDC organizer.

Keywords: mutual debt compensation; digraph of debts; Klein's cycle cancelling

\section{Introduction}

The increasing complexity of the economic environment and the large number of heterogeneous agents participating in the market necessitate a new approach in modeling creditor-debtor relationships. The seminal papers of Diamond and Dybvig [6] and Allen and Gale [2] [3] offer the graph theory as an efficient tool for modeling in this field. The subsequent boosting research performed mainly during the last decade was primarily motivated by the existing financial crises, the development of the complexity theory and the limitations of the traditional econometric techniques. The research results demonstrate a significant impact of the financial network structure on the financial risks given by the potential extent of the insolvency contagion spreading through the net. Here, Allen and Gale [3], comparing cycle and complete graph structures, conclude that the latter is more 
resilient to liquidity shocks. On the other hand, the application of more realistic network structures using different methodologies leads to ambiguous results or even controversy. For instance, Nier et al. [14] introduce the idea that "the effect of the degree of connectivity is non-monotonic, i.e. initially a small increase in connectivity increases the contagion effect; but after a certain threshold value, connectivity improves the ability of a banking system to absorb shocks." On the other hand, according to Vivier-Lirimont [19], "financial fragility clearly depends on several network characteristics. In particular, the higher the network connectivity, the larger the number of banks involved in the contagion process, and the quicker the contagion phenomenon." Despite the above-given controversies, we base our approach on the intuition that the increasing number of debt relations has a positive impact on the danger of financial insolvency. That is also a reason why the reduction of the debt relations becomes the primary goal of our article.

However, determining a way to find possibilities for debt reduction in financial networks, is not a new topic. Activities mobilizing economic agents to settle debts and cut losses from the unpaid debts have been discussed in financial research for a long time. Here, despite having many common features, the approaches differ in some details. Rotemberg [15] deals with the problem of debt repayment between ultimate lenders, ultimate borrowers and intermediaries. He considers rather consecutive repayment of the debts that is restricted by the number of transactions per time unit than debt compensation in the structure. The presence of a cycle in a debt structure represented by a directed graph demonstrates the necessity for an outer source of liquidity. Settling all the debts in the structure depends on the order of the debt repayments made by particular subjects. Rotemberg [15] is concerned with the amount of additional liquidity provided by some subjects from outside the system to settle all the obligations inside the structure. Verhoeff [18] uses graph representation to solve the problem of the settling of multiple debts between agents. The goal is to find the optimal solution with an efficient method that minimizes the number of links (transfers) between creditors and debtors and the total amount of money transferred. An important assumption about the zero total balance of transfers has been made. The efficiency of the transfer process may be expressed by the limitation that no more than $N-1$ transfers are needed in the graph with $N$ vertices ( $N$ represents the number of indebted agents). Moreover, if the cycle sub-graph exists in the structure of debts, the minimum amount of owed money can be subtracted from each graph link. Boerner and Hatfield [4] analyze the clearing process of debts between agents in the balanced (the debts vs. claims position is zero for each agent) and the unbalanced financial position. In the balanced case, the cycle removal mechanism that clears all debts is used, while in the case of unbalanced positions of agents, consecutive removal of debts in the chain is applied.

Our model is built on the aforementioned concepts; however, it differs in many important aspects and motivations. The approach we use is devoted to the 
compensation of debts among the agents. Our methodology focuses on the application of the standard graph theory algorithm to solve the debt compensation process among firms, although the problem can be rewritten as an application of the linear programming method with the minimization of debts in the given structure as an objective function. The novelty can be seen in the introduction of the following models: (i) the model maximizing the returns of the MDC organizer; (ii) the model of profitable subsidies and (iii) the model minimizing the claims exposition of the MDC organizer.

Our paper is organized as follows. In Section 2, the concept of a digraph of debts is introduced. The formalism of the mutual debt compensation (MDC) process is presented in Section 3. The standard graph approach of Klein's cycle cancelling algorithm, which solves MDC, is applied in Section 4. Modification of this algorithm leading to maximal return of the MDC organizer is described in Section 5. Sections 6 and 7 introduce the concept of a mutual debt compensation organizer in the form of a subsidy centre and the Ministry of Finance. Finally, the conclusions are presented.

\section{Digraph of Debts}

Let $V=\{1,2, \ldots, n\}$ stand for a set of firms involved in a process of mutual debt compensation. Let the debt that firm $i$ owes to firm $j$ be denoted as $y_{i, j}$. We assume that, at most, one of two mutually exclusive situations $y_{i, j}>0$ and $y_{j, i}>0$ may occur. The debtor-creditor relationships are defined by a set

$$
E=\left\{[i, j] \in V^{2} \mid y_{i, j}>0 \quad \text { as } \quad i \neq j\right\}
$$

Here, the function $y: E \rightarrow \mathrm{R}^{+}$assigns a real positive debt amount to each debtor-creditor relation. Then, using the previous structures, we may introduce formally a digraph of debts as follows:

$$
G=(V, E, y) \text {. }
$$

As set $V^{-}(i)$ denotes the in-neighborhood of vertex $i$ (representing the list of its debtors) and set $V^{+}(i)$ denotes the out-neighborhood of vertex $i$ (the list of its creditors), the receivables/debts balance of firm $i$ may be expressed as

$$
b(i)=\sum_{j \in V^{-}(i)} y_{j, i}-\sum_{j \in V^{+}(i)} y_{i, j} \quad \text { for } \quad i \in V .
$$


In this formula, the total receivables of firm $i$, represented by $\sum_{j \in V^{-}(i)} y_{j, i}$, are in part balanced by its total debts, represented by $\sum_{j \in V^{+}(i)} y_{i, j}$. It is important to note that we assume fairness of MDC. This means that the balance between the receivables and the debts expressed by Eq. (3) have to remain constant during the MDC process. Quite obviously, the debt digraph property

$$
\sum_{i=1}^{n} b(i)=0
$$

states that the total sum of receivables/debts is balanced.

\section{The MDC Process as Cycle Elimination}

This section may be perceived as an intuitive introduction to the methodology based on the cyclic properties of digraphs. The presence of a cycle in the digraph means the existence of a cyclical substructure of mutual debts in the world of interconnected firms. Let $C \subseteq G$ be a cycle in $G$ ( $G$ defined in Eq. (2)). Again, $C$ may be written as ordered triple $(V(C), E(C), y)$ comprising the set of vertices $V(C)$ together with a set $E(C)$ of arcs quantified by the debt strength described by the corresponding $y$ weights. By defining the capacity of cycle $C$ as

$$
y(C)=\min _{e \in E(C)} y_{e},
$$

the weights of the edges of $C$ may be reduced by subtracting the cycle capacity $y(C)$ from the weights of all the arcs lying in the cycle ${ }^{1}$. The assignments

$$
y_{e}:=y_{e}-y(C), \quad \forall e \in E(C)
$$

yield debt compensation since they cause a reduction in the total debts in the original digraph $G$. At the same time, the debtor-creditor relations represented by at least one arc with zero weight are removed from cycle $C$ (as well as from

Further, we are using double notation of the arcs. First, the notation $y_{i, j}$ with subscripts comprising the ordered pair $i, j$ is used if we want to stress the role of the respective vertex; second, the general notation of the $\operatorname{arc} e$ is used to indicate an element of $E$. 
digraph $G$ ) and thus the cycle is eliminated from $G$. Eq. (3) rewritten as $b(i)=\left(\sum_{j \in V^{-}(i)} y_{j, i}\right)-y(C)-\left[\left(\sum_{j \in V^{+}(i)} y_{i, j}\right)-y(C)\right]$ demonstrates that the receivables/debts balance of each firm before and after the compensation remains conserved. The graph becomes acyclical due to consecutive application of the procedure to all the cycles present in $G$.

\subsection{Example: The Change from a Cyclical to an Acyclical Graph}

Consider a simple structure of five firms with the debt structure given in Figure 1A. The debt structure creates a cycle given by a sequence of vertices and arcs 1 , $[1,2], 2,[2,3], 3,[3,4], 4,[4,5], 5,[5,1]$ (i.e. a cycle with vertices $V(C)=\{1,2,3,4,5\}$ and $\operatorname{arcs} E(C)=\{[1,2],[2,3],[3,4],[4,5],[5,1]\})$.

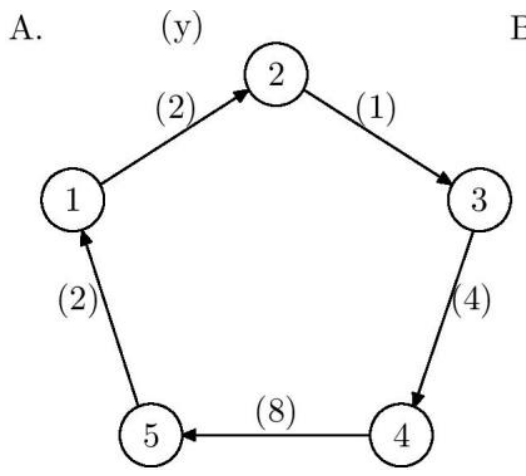

B. $\quad(\mathrm{y}-\mathrm{y}(\mathrm{C}))$

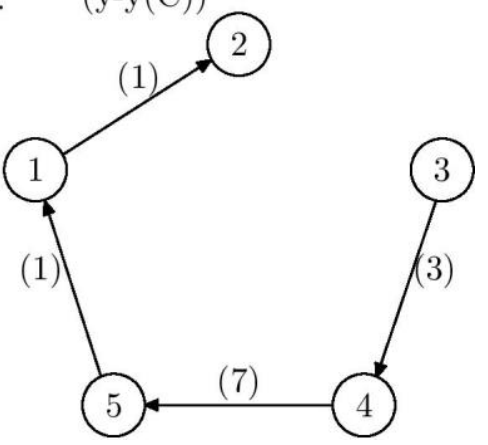

Figure 1

A) The debt structure before compensation; B) The debt structure after compensation

The minimum debt in the cycle is a debt of size 1 between 2 and 3 . The debt structure in Figure 1B is obtained by the cycle elimination, i.e. subtracting 1 from all the debts in the cycle. The resulting digraph becomes acyclical. 


\section{MDC Based on Maximum Circulation in the Digraph of Debts}

Consider a strongly connected digraph $G=(V, E, y)^{2}$. In this graph, we define a circulation function [see [7] p. 146] $x: E \rightarrow \mathrm{R}_{0}^{+}$, which satisfies inequality

$0 \leq x_{i, j} \leq y_{i, j}$

and constraint

$$
\sum_{j \in V^{-}(i)} x_{j, i}=\sum_{j \in V^{+}(i)} x_{i, j}, \quad i \in V .
$$

Further, we will demonstrate the suitability of the circulation concept for MDC modeling. The variable $x_{i, j}$ is interpreted as part of a debt $y_{i, j}$, which may be compensated between firms $i$ and $j$. Therefore, the total amount of debts compensated due to the pair-wise effect of circulation may be written as follows:

$$
\sum_{[i, j] \in E} x_{i, j}
$$

In the MDC process, the receivables/debts balance of any firm, must not be worsened or improved. The relation in Eq. (8) reports the compensation of the receivables and debts on the level of single firm $i$. Therefore, it is quite obvious to expect that respecting this condition should not violate the balance of the receivables/debts. Assume that before the compensation process starts, we have $b(i)$ expressed in the form of Eq. (3). Subsequently, adding zero in the form of the right-hand side minus the left-hand side of Eq. (8) leaves $b(i)$ unchanged:

$$
b(i)=\sum_{j \in V^{-}(i)}\left(y_{j, i}-x_{j, i}\right)-\sum_{j \in V^{+}(i)}\left(y_{i, j}-x_{i, j}\right) \text {. }
$$

The original debts $y_{i, j}$ are now expressed in the compensated form. From this it follows that the minimization of debts becomes equivalent to the identification of maximal circulation

$$
\max _{x} \sum_{[i, j] \in E} x_{i, j}
$$

2 The digraph $G=(V, E, y)$ is strongly connected if for every pair of vertices $i, j \in V$ a direct path exists from $i$ to $j$ as well as from $j$ to $i$. 
subject to the constraints expressed by Eq. (7) and Eq. (8). The main reason why we decided to pass to the maximum circulation problem is that it can be tackled by means of the standard linear programming methods, as well as, standardized special-purpose graph algorithms. For example, the application of Klein's cycle cancelling (see [12]), originally applied to find the minimum cost circulation, might be possible ${ }^{3}$. The algorithm is based on the identification of all the cycles and the determination of the optimal sequence of the elimination in the digraph respecting the trade-off among the cycle capacities (see [7]). Based on Eq. (7), we can conclude that if $y_{i, j}=0$ then $x_{i, j}=0$. Thus, the total debt minimization does not cause the creation of new creditor debtor relations, which is considered as an important aspect of the proposed model.

\subsection{Example: Cycle Cancelling - from a Cyclical to an Acyclical Graph}

Figure 2 describes the mutual debts of five firms. The total amount of mutual debts is equal to the sum of arc weights, i.e. $\$ 25$ mil. There are three cycles in the digraph. The first one is given by the debt sequence 1, [1,2], 2, [2,3], 3, [3,4], 4, $[4,5], 5,[5,1], 1$, the second one by the sequence $1,[1,2], 2,[2,5], 5,[5,1], 1$ and the third one by the sequence $1,[1,3], 3,[3,4], 4,[4,5], 5,[5,1], 1$. All these cycles contain a common arc $[5,1]$, which determines the minimum capacity in the second and the third cycle. Consequently, the elimination of the second cycle causes the elimination of arc [5,1] and thus the elimination of the first and third cycles, too. On the other hand, the elimination of the third cycle causes the elimination of the first and the second cycle (see Figure 3). In both approaches, the digraph becomes acyclical, meaning that no more debts may be compensated by the cycle cancelling. The total amounts of uncompensated debts are different in the two cases (see Figure 3A, Figure 3B) - \$19 mil. in the first case and $\$ 17$ mil. in the second one. It is quite obvious that the most appropriate method is to eliminate cycle $1,[1,2], 2,[2,3], 3,[3,4], 4,[4,5], 5,[5,1], 1$ first and then to eliminate $1,[1,3], 3,[3,4], 4,[4,5], 5,[5,1], 1$. We can see that elimination applied in a suitable order causes the total amount of the uncompensated debts to remain at the level of $\$ 16$ mil. (see Figure 4).

3 We choose Klein's algorithm for demonstrative purposes; more efficient circulation algorithms were proposed later (Goldberg and Tarjan [10] is a good example). 


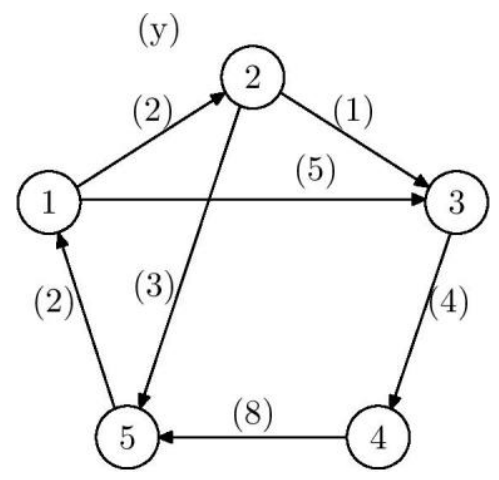

Figure 2

The structure of debts before their compensation

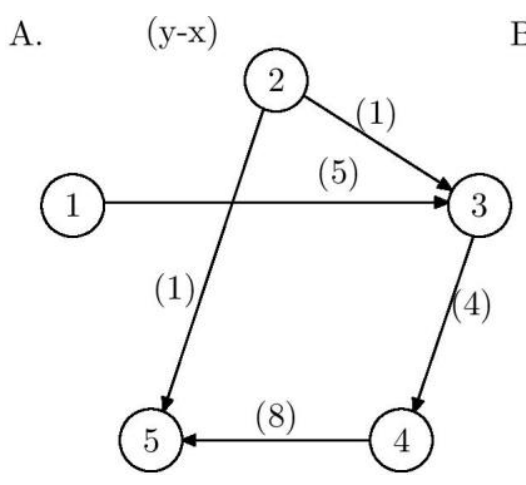

B. $(\mathrm{y}-\mathrm{x})$

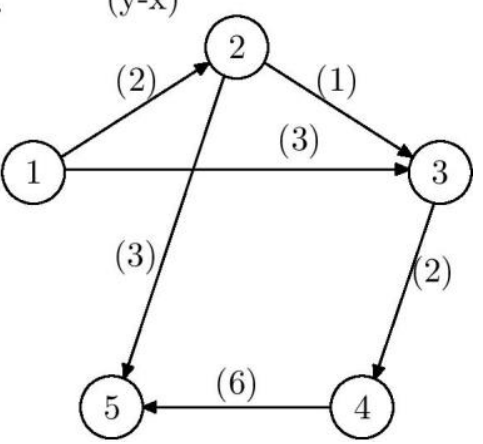

Figure 3

The remaining debt structure if: A) cycle 1, [1,2], 2, [2,5], 5, [5,1], 1 is eliminated; B) cycle 1, [1,3], 3, $[3,4], 4,[4,5], 5,[5,1], 1$ is eliminated

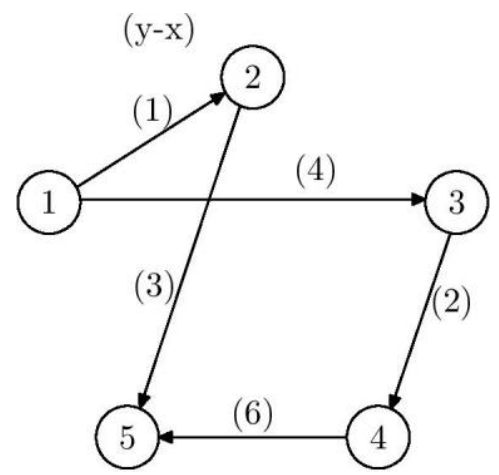

Figure 4

The structure of debts that stems from the application of cycle cancelling 
The above-formulated MDC heuristic is rather intuitive and does not provide the optimal ordering of the cycle elimination. That is why it is inapplicable in the case of large debt networks with a high level of density. Here, we decided to maximize the graph circulation by the modified Klein's cycle-cancelling method (see [12]), which is extremely effective in enabling us to solve the extended graph problems. However, we avoid the usage of large graphs in this article because our pictures are intended to be easy to understand. We focus mainly on the economic aspects of the debt problems. We did not want to spend too much time on some of the finer details of the standard algorithm.

\section{Return of the Mutual Debt Compensation Organizer}

Companies themselves are mostly unable to solve the problem of debts, which needs extensive multi-company projects that can help to solve the problem by initiating and organizing the process of debt compensation. In practice, the organizer of MDC expects to be rewarded. Its return function $c: E \rightarrow\langle 0 ; 1\rangle$ assigns a unit price per monetary unit of each compensated debt. More detailed information may be captured by digraph $G=(V, E, y, c)$. The total income of the organizer can be written as

$\sum_{[i, j] \in E} c_{i, j} x_{i, j}$

The maximal return due to application of the circulation may be defined as follows:

$\max _{x} \sum_{[i, j] \in E} c_{i, j} x_{i, j}$

Again, the optimization is considered in common with the constraints defined by Eq. (7) and Eq. (8).

\section{The Subsidy Center and the Problem of Restricted Subsidies}

In practice, there are a few examples in which a non-participating subject enters a debt structure to endow a highly indebted firm. It enables it to cover its debts, which consequently allows the elimination of the whole chain of induced debts caused by the insolvency of a highly indebted firm. Now, we formulate a model in 
which the subsidy centre does not know a priori which firm should be subsidized. Conversely, the list of firms will be determined by the algorithm. In addition, we assume that the sources available for providing the subsidies are restricted. The vertex $(n+1)$ is added to substitute the effect of the subsidy centre. All of its relations will be represented by $[n+1, i], i \in V$ arcs. Assuming this structure, the linear programming model from Eq. (13) takes the form

$$
\begin{aligned}
& \max _{x}\left[\sum_{[i, j] \in E} c_{i, j} x_{i, j}-\sum_{i \in V} x_{n+1, i}\right], \\
& \sum_{j \in V^{-}(i)} x_{j, i}-\sum_{j \in V^{+}(i)} x_{i, j}=0 \quad \text { for } \quad i \in V=\{1,2, \ldots, n\}, \\
& \sum_{i \in V} x_{n+1, i} \leq B, \\
& 0 \leq x_{i, j} \leq y_{i, j} \quad \text { for }[i, j] \in E \\
& 0 \leq x_{n+1, i} \leq y_{n+1, i} \quad \text { for } \quad i \in V .
\end{aligned}
$$

According to Eq. (16), the total amount of the subsidies provided is restricted by constant $B$. The subsidy provided to the $i^{\text {th }}$ firm is restricted by its upper bound, expressed by Eq. (18). Now, we finish the specific MDC formulation for the purposes of linear programming. This approach is, however, less efficient for the solving of many vertex graph problems. Greater efficiency may be gained by Klein's cycle-cancelling method. In the next section, we will discuss its adaption to the given problem.

\subsection{Klein's Cycle Cancelling Adapted to the Model of the Subsidy Centre}

Vertex $(n+1)$ does not include any incident input edges; no circulation through this vertex is possible. As a solution, we propose a modified debt digraph $\bar{G}=(\bar{V}, \bar{E}, \bar{y}, \bar{c})$ with modified topology including subsidy vertex $(n+1)$ as well as auxiliary vertex 0 . Formally,

$$
\bar{V}=V \cup\{n+1\} \cup\{0\} \text {. }
$$

All the edges $\{[n+1, i], \forall i \in V\}$ weighted by $y_{n+1, i}$ represent the possible support provided by the subsidy centre localized at the vertex $(n+1)$. The arc $[0, n+1]$ enables us to create the flow condition (see constraint Eq. (8)) 
balancing the subsidy centre. Vertex 0 is balanced via additional edges $\{[i, 0], \forall i \in V\}$. Then, the edges of $\bar{G}$ may be specified as follows:

$\bar{E}=E \cup\{[i, 0] \mid i \in V\} \cup\{[0, n+1]\} \cup\{[n+1, i] \mid i \in V\}$.

We now specify function $\bar{y}: \bar{E} \rightarrow \mathrm{R}^{+}$, which consists of the additional rules with respect to the original $y$

$$
\begin{aligned}
& \bar{y}_{i, j}=y_{i, j} \quad \text { for } \quad[i, j] \in E, \\
& \bar{y}_{i, 0}=y_{n+1, i} \quad \text { for } \quad i \in V, \\
& \bar{y}_{0, n+1}=\min \left(B, \sum_{i \in \bar{V}^{-}(n+1)} y_{n+1, i}\right), \\
& \bar{y}_{n+1, i}=y_{n+1, i} .
\end{aligned}
$$

Eq. (21) shows that the distribution of debts remains the same as in the original debt digraph $G$, while Eq. (23) describes the total amount of disposable subsidies. The remaining constituent of $\bar{G}$ represents the return $\bar{c}: \bar{E} \rightarrow \mathrm{R}$. The costs of debt reduction per monetary unit are expressed by $\bar{c}_{n+1, i}$

$$
\begin{aligned}
& \bar{c}_{i, j}=c_{i, j} \quad \text { for } \quad[i, j] \in E, \\
& \bar{c}_{i, 0}=0 \quad \text { for } \quad i \in V, \\
& \bar{c}_{0, n+1}=0, \\
& \bar{c}_{n+1, i}=-1 \quad \text { for } \quad i \in V .
\end{aligned}
$$

After the above modifications, we obtain digraph $\bar{G}$, which enables the application of the cycle-cancelling algorithm for the maximum return circulation.

\subsection{Example: The Role of the Subsidy Centre}

The structure of the mutual debts among the firms is represented in the digraph in Figure 2. We considered $\$ 25$ mil of the total mutual debts. The coefficients $c_{i, j}$ are set to be equal to 0.5 for all the arcs' returns. We assume that the subsidy centre $((n+1)=$ vertex6 $)$ is allowed to provide a subsidy by an amount equal 
to $\$ 2$ mil. to the firms $1,3,5$. The total sum of the subsidies is restricted by the upper bound $B=\$ 3$ mil. The diagram of this situation is presented in Figure 5 .

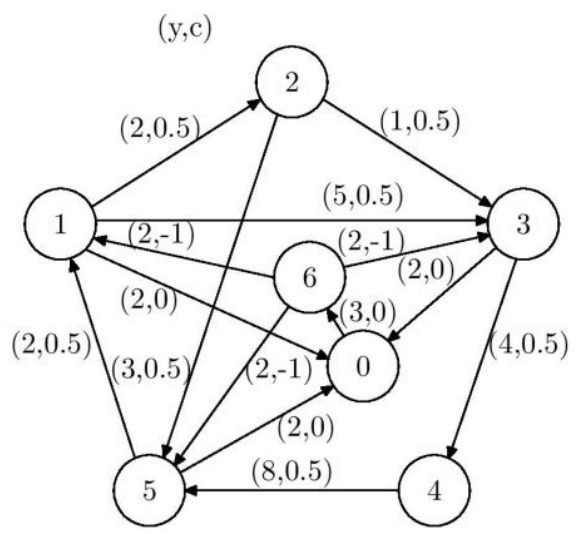

Figure 5

The initial situation before the compensation of debts

The optimal solution of the above-mentioned optimization model is given in Figure 6A. The sum of the compensated debts is $\$ 15$ mil. The subsidy center provides a subsidy to firm 1 to the amount of $\$ 2$ mil. The residual debts are represented in Figure 6B. It shows that the subsidies available were not completely used and $\$ 1$ mil. remained. The MDC organizer achieves a profit of $\$ 5.5$ mil.

A.

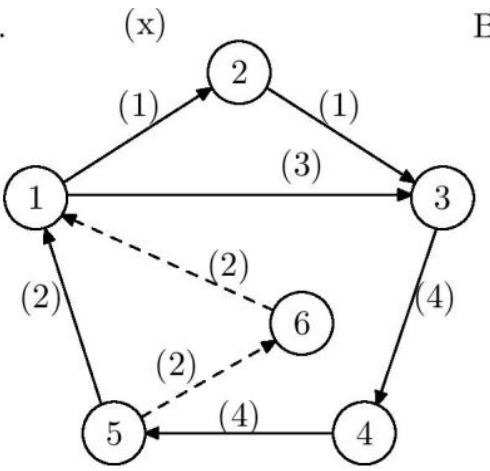

B. $(\mathrm{y}-\mathrm{x})$

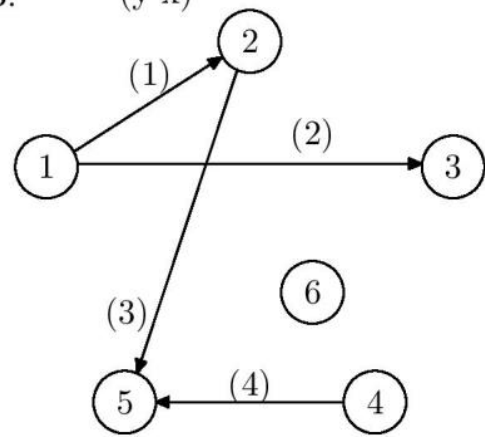

Figure 6

A) The circulation of compensated debts; B) The structure of mutual debts that results from the process of their compensation 


\section{Minimization of Receivables by the Ministry of Finance}

The organizer of the mutual debt compensation is unlikely to be interested in diminishing the total debts in the whole complicated structure of debts; instead, he claims to decrease the receivables/debts of a single particular subject. This motivated us to consider a realistic situation, in which the role of the organizer of debt elimination is the Ministry of Finance. On one hand, the Ministry collects its income from firms (e.g. by way of taxes), but on the other hand it spends its budget by carrying payments to the firms providing services. Here we do not pay attention to the financial relations with another subjects. Let $V=\{1,2, \ldots, n\}$ be a set of firms. By formulation in terms of graphs, the Ministry of Finance, represented by vertex $n+1$, is intended for mutual debt compensation. Then, a set $\bar{V}=V \cup\{n+1\}$ involves all the subjects included in the compensation process. Let $y_{i, n+1}$ represent a debt of firm $i$ towards the Ministry and $y_{n+1, j}$ represent the receivable of firm $j$ towards the Ministry. The comprehensive debt structure is represented by

$$
\begin{gathered}
\bar{E}=E \cup\left\{[n+1, j] \mid j \in V, y_{n+1, j}>0\right\} \cup \\
\cup\left\{[i, n+1] \mid i \in V, y_{i, n+1}>0\right\},
\end{gathered}
$$

where the structure of debts $E$ is defined in Section 2. The function $\bar{y}$ assigning a particular debt to each debt relation is defined as follows:

$\bar{y}: \bar{E} \rightarrow \mathrm{R}^{+}$,

where

$$
\begin{aligned}
& \bar{y}_{i, j}=y_{i, j} \quad \text { for } \quad[i, j] \in E, \\
& \bar{y}_{i, n+1}=y_{i, n+1} \quad \text { for } \quad i \in V, \\
& \bar{y}_{n+1, j}=y_{n+1, j} \quad \text { for } \quad j \in V .
\end{aligned}
$$

The digraph $\bar{G}=(\bar{V}, \bar{E}, \bar{y})$ represents the mutual debt structure among the firms and the Ministry. The receivables/debts balance of the Ministry is expressed as

$\sum_{i \in \bar{V}^{-}(n+1)} \bar{y}_{i, n+1}-\sum_{j \in \bar{V}^{+}(n+1)} \bar{y}_{n+1, j}=b(n+1)$. 
By following the ideas introduced above, we define circulation

$$
\begin{aligned}
& x: \bar{E} \rightarrow \mathrm{R}^{+} ; \quad 0 \leq x_{i, j} \leq \bar{y}_{i, j} ; \quad i \in \bar{V}, \\
& \sum_{j \in \bar{V}^{-}{ }_{(i)}} x_{j, i}=\sum_{j \in \bar{V}^{+}(i)} x_{i, j},
\end{aligned}
$$

as mutual debt compensation given in a digraph of debts. It should be noted that if the receivables/debts balance of subjects $1,2, \ldots, n$ in digraph $\bar{G}=(\bar{V}, \bar{E}, \bar{y})$ remains constant in the process of the mutual debt compensation, then the balance of subject $n+1$ remains constant, too. This prevents the Ministry from improving its receivables/debts balance by organizing the mutual debt compensation. The proof is based on the fact that the sum of the receivables/debts balances of all the subjects before debt compensation is (see Section 3)

$$
\sum_{i \in \bar{V}}\left(\sum_{j \in \bar{V}^{-}(i)} \bar{y}_{j, i}\right)-\sum_{i \in \bar{V}}\left(\sum_{j \in \bar{V}^{+}(i)} \bar{y}_{i, j}\right)=0 .
$$

The balance condition must also be satisfied after the compensation, i.e. for

$$
\begin{aligned}
& \sum_{i \in V}\left(\sum_{j \in \bar{V}^{-}(i)}\left(\bar{y}_{j, i}-x_{j, i}\right)-\sum_{i \in V}\left(\sum_{j \in \bar{V}^{+}(i)}\left(\bar{y}_{i, j}-x_{i, j}\right)\right)+\right. \\
& +\sum_{i \in \bar{V}^{-}(n+1)}\left(\bar{y}_{i, n+1}-x_{i, n+1}\right)- \\
& -\sum_{i \in \bar{V}^{+}(n+1)}\left(\bar{y}_{n+1, i}-x_{n+1, i}\right)=0 .
\end{aligned}
$$

If the fairness condition of the compensated debts is satisfied for each of the $n$ participating firms

$$
\sum_{j \in \bar{V}^{-}(i)} x_{j, i}=\sum_{j \in \bar{V}^{+}(i)} x_{i, j},
$$

the following condition must hold, too

$$
\sum_{i \in V} \sum_{j \in \bar{V}^{-}(i)} x_{j, i}=\sum_{i \in V} \sum_{j \in \bar{V}^{+}(i)} x_{i, j} .
$$

It directly implies the fairness of the compensation of the Ministry of Finance:

$$
\sum_{i \in \bar{V}^{-}(n+1)} x_{i, n+1}=\sum_{i \in \bar{V}^{+}(n+1)} x_{n+1, i} .
$$


The problem of the minimization of Ministry receivables may be modeled as the maximization of the objective function:

$$
\begin{aligned}
& \max \sum_{i \in \bar{V}^{-}(n+1)} x_{i, n+1} \\
& \sum_{j \in \bar{V}^{-}(i)} x_{j, i}=\sum_{j \in \bar{V}^{+}(i)} x_{i, j} \quad \text { for } \quad i \in \bar{V}, \\
& 0 \leq x_{i, j} \leq \bar{y}_{i, j} \quad \text { for } \quad[i, j] \in \bar{E} .
\end{aligned}
$$

Such formulation admits the application of the linear programming methods. They are efficient for graphs of a small or moderate size. On the other hand, Klein's cycle-cancelling method, discussed in the next subsection, represents a more suitable tool for larger graphs.

\subsection{Receivables of the Ministry of Finance Tackled by Klein's Cycle-Cancelling Method}

The implementation of new virtual vertex 0 in order to apply Klein's cyclecancelling method yields

$$
\tilde{V}=\bar{V} \cup\{0\}
$$

Then, all the arcs entering vertex $(n+1)$ are redirected to the virtual vertex 0 , i.e.

$$
\left\{[i, n+1] \mid i \in V ; y_{i, n+1}>0\right\} \rightarrow\{[i, 0] \mid i \in V\}
$$

and a new arc $[0, n+1]$ is added. It yields the set of arcs

$$
\begin{gathered}
\tilde{E}=E \cup\left\{[i, 0] \mid i \in V, y_{i, n+1}>0\right\} \cup\{[0, n+1]\} \cup \\
\cup\left\{[n+1, i] \mid i \in V, y_{n+1, i}>0\right\} .
\end{gathered}
$$

The function $\tilde{y}: \tilde{E} \rightarrow \mathrm{R}^{+}$is defined as

$$
\begin{aligned}
& \tilde{y}_{i, j}=y_{i, j} \quad \text { for } \quad[i, j] \in E, \\
& \tilde{y}_{i, 0}=y_{i, n+1} \quad \text { for } \quad i \in \bar{V}^{-}(n+1), \\
& \tilde{y}_{0, n+1}=\sum_{i \in \bar{V}^{-}(n+1)} y_{i, n+1},
\end{aligned}
$$


$\tilde{y}_{n+1, i}=y_{n+1, i} \quad$ for $\quad i \in V$.

Due to the fact that digraph $\tilde{G}=(\tilde{V}, \tilde{E}, \tilde{y})$ contains vertex 0 , the minimization of the receivables becomes equivalent to the finding circulation with the maximum value along arc $[0, n+1]$. This leads us to the application of the aforementioned maximum return circulation method with the return function elements

$\tilde{c}_{i, j}=0 \quad$ for $\quad[i, j] \in E$,

$\tilde{c}_{i, 0}=0 \quad$ for $\quad i \in V$,

$\tilde{c}_{0, n+1}=1$,

$\tilde{c}_{n+1, i}=0 \quad$ for $\quad i \in V$.

\subsection{Example: The Ministry of Finance as the MDC Organizer}

Let the initial situation of mutual debts be represented by the digraph in Figure 7A. The vertex enumerated by 6 represents the Ministry of Finance. The Ministry has receivables towards firm 5 to an amount of $\$ 2$ mil. and towards firm 4 to an amount of $\$ 3$ mil. The Ministry owes $\$ 5$ mil. to firm 1 and $\$ 1$ mil. to firm 3. We assume that the Ministry organizes the compensation of mutual debts whereby the minimizing of its receivables is its obvious objective function (see Eq. (42)).
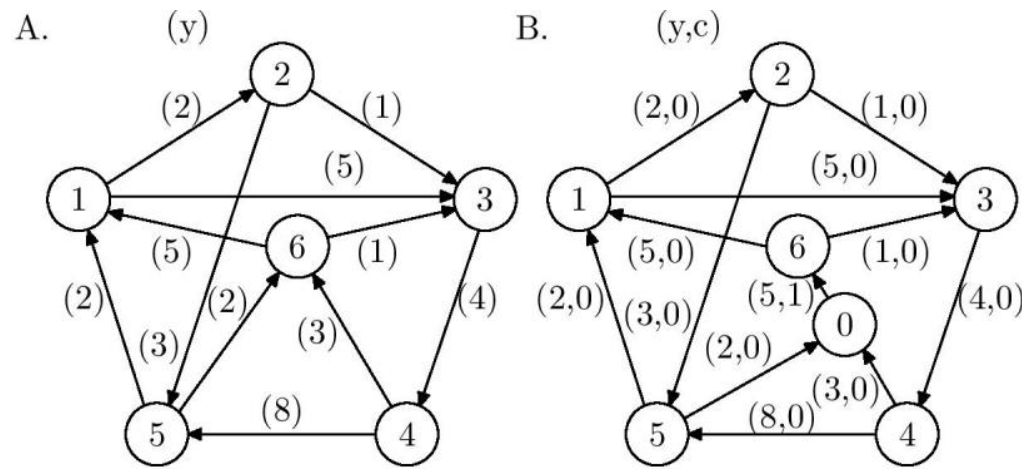

Figure 7

The initial situation before debt compensation

A) $\bar{G}=(\bar{V}, \bar{E}, \bar{y})$ to be solved by the linear programming methods

B) $\widetilde{G}=(\tilde{V}, \tilde{E}, \tilde{y})$ to be solved by an appropriate cycle-cancelling method 
In order to enable the application of the circulation algorithm, we extend the digraph that includes vertex 0 (see Figure 7B). Accordingly, the arcs $[4,6]$ and $[5,6]$ with weights 2 and 3 in Figure 7A are redirected to the 0 (see Figure 7B). In addition, a new arc $[0,6]$ with debt weight 5 is created. This modified digraph becomes ready for the application of the circulation maximization along $[0,6]$. After the application of the cycle-cancelling method, the residual debts diminish to 14 (see Figure 8B). All the cycles are eliminated from the final form of the digraph.
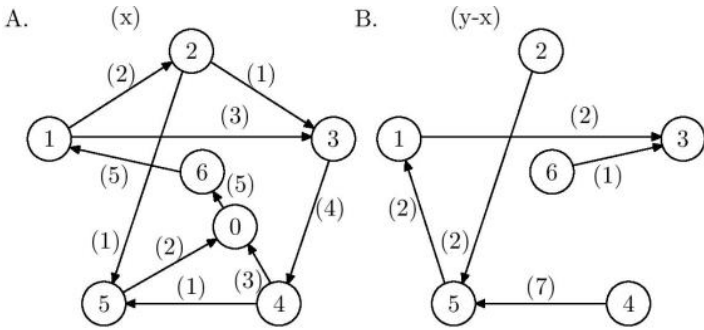

Figure 8

A) The circulation of the compensated debts; B) The resulting structure of debts

\section{Conclusion}

In this paper, we discuss the principles of the graph theory that are relevant to performing the MDC procedure. Principally, new formulations are presented, including models with a subsidy center and their conversion into a form tractable by Klein's cycle-cancelling algorithm. Besides the solving of the general task of the cycle cancelling, we demonstrate the method's applicability to the problem of receivables of a single selected firm. Our research shows that both topic and methodology are still far from being exhausted. The approach can be adapted straightforwardly to digraphs of the International relationships among the countries-vertices equipped with the import-export links. The impulses for ongoing research may come from studies on the altering nature of the economic world. Dynamic graph models of debt structures should be introduced that formalize the concept of the flexible links. These can be transformed under the influence of the stochastically varying external conditions represented by the reevaluating/devaluating domestic currency.

\section{Acknowledgement}

The paper was elaborated within the project VEGA No 1/1195/12.

\section{References}

[1] Ahuja R. K., Magnanti T. L., Orlin J. B.: Network Flows: Theory, Algorithms, and Applications. Prentice Hall, University of Michigan (1993)

[2] Allen F., Gale D.: Optimal Financial Crises. Journal of Finance (1998), 4: 1245-1284 
[3] Allen F., Gale D.: Financial Contagion. Journal of Political Economy (2000), 1: 1-33

[4] Boerner L., Hatfield J. W.: The Economics of Debt Clearing Mechanisms. Working Paper No. 2010/27, School of Business, Economics Discussion Paper (2010)

[5] Chartrand G., Oellermann O. R.: Applied and Algorithmic Graph Theory. McGraw-Hill, University of California (1993)

[6] Diamond D. W., Dybvig P. H.: Bank Runs, Deposit Insurance, and Liquidity. Journal of Political Economy (1983), 5: 401-419

[7] Diestel R.: Graph Theory. Springer-Verlag, London (1997)

[8] Fecenko J.: About Optimisation of Receivables and Payables Compensation. Ekonomický časopis (1994), 42: 360-374

[9] Gazda V.: Mutual Debts Compensation as Graph Theory Problem. In: Mathematical Finance (2001)

[10] Goldberg A. V., Tarjan R. E.: Finding Minimum-Cost Circulations by Canceling Negative Cycles. Journal of the ACM (1989), 36(4): 873-886

[11] Harary F.: Graph Theory. Addison-Wesley Publishing Company, London (1969)

[12] Klein M.: A Primal Method for Minimum Cost Flow with Application to the Assignment and Transportation Problem. Management Science (1967), 14(3): 205-220

[13] Jun Q., Wing L. N.: Asymmetric Liquidity Risk Premia in Intraday High Frequency Trading. International J. of Applied Mathematics (2010), 40(1)

[14] Nier E., Yang J., Yorulmazer T., Alentorn A.: Network Models and Financial Stability. Journal of Economic Dynamics and Control (2007), 31(6): 2033-2060

[15] Rotemberg J. J.: Liquidity Needs in Economies with Interconnected Financial Obligations. National Bureau of Economic Research, Working Paper (2008)

[16] Tarjan R. E.: Depth First Search and Linear Graph Algorithms. SIAM J. Computing (1972), 1: 146-160

[17] Upper C.: Simulation Methods to Assess the Danger of Contagion in Interbank Markets. Journal of Financial Stability (2011), 7(1): 111-125

[18] Verhoeff T.: Settling Multiple Debts Efficiently: An Invitation to Computing Science. Informatics in Education (2004), 3(1): 105-126

[19] Vivier-Lirimont S.: Contagion in Interbank Debt Networks. Working Paper (2006) 